\title{
Some $A$-Stable Methods for Stiff Ordinary Differential Equations
}

\author{
By R. K. Jain
}

\begin{abstract}
This paper gives some $A$-stable methods of order $2 n$, with variable coefficients, based on Hermite interpolation polynomials, for the stiff system of ordinary differential equations, making use of $n$ starting values. The method is exact if the problem is of the form $y^{\prime}(t)=P y(t)+Q(t)$, where $P$ is a constant and $Q(t)$ is a polynomial of degree $2 n$.
\end{abstract}

1. Introduction. Many physical problems lead to ordinary differential equations with a property given by the following definition:

Definition 1. A system of ordinary differential equations $y^{\prime}(t)=f(t, y), y(a)=y$ is said to be stiff if the eigenvalues of the matrix $\partial f(t, y) / \partial y$ have negative real parts at every time $t$ and differ greatly in magnitude.

A linear $k$-step method with constant coefficients for the numerical solution of ordinary differential equations is given by

$$
\sum_{i=0}^{k} \alpha_{i} y_{n+i}=h \sum_{i=0}^{k} \beta_{i} y_{n+i}^{\prime}, \quad \text { where } \alpha_{k} \neq 0 \text { and }\left|\alpha_{0}\right|+\left|\beta_{0}\right|>0 .
$$

Stiff equations present a difficulty in numerical integration, since the integration interval is determined by the fastest rate and the region of integration is determined by the slowest rate. Conventional methods of the form (1.1) are unstable if the step size used is much greater than the smallest time constant. Dahlquist [1] introduced the concept of $A$-stability in connection with the integration of stiff systems of differential equations. $A$-stability is defined as:

Definition 2. A $k$-step method is called $A$-stable if all the solutions of (1.1) tend to zero as $n \rightarrow \infty$, when the method is applied with fixed positive $h$ to any differential equation of the form $d y / d t=\lambda y$, where $\lambda$ is a complex constant with negative real part.

Dahlquist [1] has also shown that if (1.1) is to be stable for all $\lambda$, such that $\operatorname{Re}(h \lambda) \leqq 0$, then the order of the method cannot exceed two. He also showed that the best method in this sense is the trapezoidal rule. In order to achieve greater accuracy and order, we study nonlinear methods (see Treanor [2]) or methods with variable coefficients. Norsett [3] derived a class of $A$-stable methods with variable coefficients of order $n$ using Lagrangian interpolation polynomials passing through the $n$ starting values.

In this paper, we have derived an integration method of order $2 n$ with variable

Received September 9, 1970, revised November 30, 1970.

AMS 1970 subject classifications. Primary 65L05.

Key words and phrases. Stiff system of ordinary differential equations, $A$-stability, multi-step methods, Lagrangian interpolation polynomials, Hermite interpolation polynomials.

Copyright @ 1972, American Mathematical Society 
coefficients using Hermite interpolation polynomials passing through the $n$ starting values. A proof that the present method is $A$-stable in the sense of Dahlquist [1] is included in Section 3.

2. Derivation of the Method. Let the problem be defined by the equation

$$
y^{\prime}(t)=f(t, y), \quad y(a)=y
$$

over the interval $I=[a, b]$. Choose $t_{n} \in I$ and define $t_{n-i}=t_{n}-i h$. It is assumed that the solution of (2.1) is known at the $n$ starting values $(i=0,1, \cdots, n-1)$.

Instead of considering the equation $y^{\prime}(t)=f(t, y)$, we consider the function $y^{\prime}(t)+P y(t)$. The main steps in the argument are as follows:

1. Approximate the function $y^{\prime}(t)+P y(t)$ by the Hermite interpolation polynomial $T(t)$ at the points $t_{n-i}, i=0,1, \cdots, n-1$.

2. Integrate the exact differential equation $y^{\prime}(t)+P y(t)=T(t)$ between the limits $t_{n}$ to $t_{n+1}$.

3. Choose $P$ as an approximation to $-(\partial f / \partial y)_{n}$.

4. Determine the order of the method and show that it is $A$-stable.

Thus approximating the function $y^{\prime}(t)+P y(t)$ by the Hermite interpolation polynomial, we obtain

$$
y^{\prime}(t)+P y(t)=\sum_{i=1}^{n} h_{i}(t)\left(f_{i}+P y_{i}\right)+\sum_{i=1}^{n} h_{i}(t)\left(f_{i}^{\prime}+P f_{i}\right)+T E,
$$

where $h_{i}(t)$ and $\bar{h}_{i}(t)$ are the standard Hermite functions of degree $2 n$ (see Ralston $[4$, p. 62] $), f_{i}=f\left(t_{i}, y_{i}\right)$ and $f_{i}^{\prime}=f^{\prime}\left(t_{i}, y_{i}\right)$.

$$
T E=\frac{1}{2 n !} F^{(2 n)}(\xi) \pi^{2}(t), \quad \text { where } F(t)=f(t)+P y(t),
$$

and $a<\xi<b$.

Obviously, (2.2) is an exact differential equation. Integrating (2.2) from $t_{n}$ to $t_{n+1}$, we obtain

$$
y_{n+1}=\bar{e}^{P h} y_{n}+\bar{e}^{P t_{n+1}}\left[\sum_{i=1}^{n}\left(H_{i} F_{i}+\bar{H}_{i} F_{i}^{\prime}\right)\right]+R_{n},
$$

where

$$
\begin{gathered}
H_{i}=\int_{t_{n}}^{t_{n+1}} e^{P t} h_{i}(t) d t, \quad \bar{H}_{i}=\int_{t_{n}}^{t_{n+1}} e^{P t} h_{i}(t) d t, \\
R_{n}=\frac{\bar{e}^{P t_{n+1}}}{(2 n) !} \int_{t_{n}}^{t_{n+1}} e^{P t} F^{(2 n)}(\xi) \pi^{2}(t) d t .
\end{gathered}
$$

Since $P$ is an approximation to $-(\partial f / \partial y)_{n}$ for a single differential equation, a natural choice of $P$ is

$$
P=-\frac{f_{n}-f\left(t_{n}, y_{n-1}\right)}{y_{n}-y_{n-1}} .
$$

For a system of equations, the choice of $P$ may depend on the efficiency of the routine to evaluate $e^{-P h}$, where $P$ is a matrix. A simple choice of $P$ is the diagonal matrix 


$$
P_{i i}=-\frac{f_{n}^{i}-f^{i}\left(t_{n}, y_{n}^{1}, \cdots, y_{n-1}^{i}, \cdots, y_{n}^{i}\right)}{y_{n}^{i}-y_{n-1}^{i}} .
$$

3. The $A$-Stability and Order of the Method (2.3). We shall show here that (2.3) is of order $2 n$ and is $A$-stable.

THEOREM. The method (2.3) is A-stable and of order $2 n$ with the usual definition of $A$-stability given by Dahlquist [1].

Proof. Let $f(t, y)=\lambda y$, where $\lambda$ is a complex constant and $\operatorname{Re} \lambda<0$. Thus, $P=-\lambda$. Obviously, $F_{i}=f_{i}+P y_{i}=\lambda y_{i}-\lambda y_{i}$ is zero for every $i$. Similarly, $F_{i}^{\prime}=f_{i}^{\prime}+P f_{i}=\lambda y_{i}^{\prime}-\lambda y_{i}^{\prime}$ is also zero for every $i$. Hence, (2.3) reduces to $y_{n+1}=$ $e^{\lambda h} y_{n}$. Since $\operatorname{Re} \lambda<0, y_{n} \rightarrow 0$ as $n \rightarrow \infty$ for any fixed $h$ and hence (2.3) is $A$-stable.

Taking $s=\left(t-t_{n}\right) / h,(2.3)$ can be rewritten as

$$
y_{n+1}=\bar{e}^{P h} y_{n}+h \bar{e}^{P h}\left[\sum_{i=1}^{n}\left(H_{i} F_{i}+\bar{H}_{i} F_{i}^{\prime}\right)\right]+R_{n} \text {, }
$$

where

$$
\begin{aligned}
H_{i}=\int_{0}^{1} e^{P h s} h_{i}(s) d s, \quad \bar{H}_{i}=\int_{0}^{1} e^{P h s} h_{i}(s) d s, \quad i=1,2, \cdots, n, \\
R_{n}=\frac{h^{2 n+1}}{2 n !} \bar{e}^{P h} \int_{0}^{1} e^{P h s} F^{(2 n)}(\xi) \pi^{2}(s) d s \\
=\frac{h^{2 n+1}}{2 n !} \bar{e}^{P h} \int_{0}^{1} F^{(2 n)}(\xi) \pi^{2}(s) d s+O\left(h^{2 n+2}\right) \\
=\frac{h^{2 n+1}}{2 n !} \bar{e}^{P h} F^{(2 n)}(\xi) \int_{0}^{1} \pi^{2}(s) d s+O\left(h^{2 n+2}\right) \\
=h^{2 n+1} \bar{e}^{P h} F^{(2 n)}(\xi) \Lambda_{n}+O\left(h^{2 n+2}\right),
\end{aligned}
$$

where $\Lambda_{n}=(1 / 2 n !) \int_{0}^{1} \pi^{2}(s) d s$ and hence the method (2.3) is of order $2 n$.

4. A Few Particular Cases. If we look at (3.1), we find that the integrations involved to determine $H_{i}, H_{i}$ and $R_{n}$ are of the form

$$
I_{n}=\int_{0}^{1} e^{P h s}\left(\sum_{i=1}^{N} A_{i} s^{i}\right) d s,
$$

where $N$ is a positive integer depending on $n$. The coefficients $H_{i}$ and $\vec{H}_{i}$ are of the form

$$
\begin{aligned}
& H_{i}=\sum_{r=1}^{2 n} a_{r}\left(\frac{1}{P h}\right)^{r} e^{P h}+h \sum_{r=1}^{2 n} b_{r}\left(\frac{1}{P h}\right)^{r}, \\
& H_{i}=\sum_{r=1}^{2 n} \alpha_{r}\left(\frac{1}{P h}\right)^{r} e^{P h}+h \sum_{r=1}^{2 n} \beta_{r}\left(\frac{1}{P h}\right)^{r} .
\end{aligned}
$$

Taking a simple case $n=1$, we have

$$
\begin{array}{lll}
h_{1}(t)=1 ; & h_{1}(t)=t-t_{1} ; & \pi(t)=t-t_{1} \\
h_{1}(s)=1 ; & h_{1}(s)=s ; & \pi(s)=s ;
\end{array}
$$




$$
\begin{aligned}
& H_{1}=\int_{0}^{1} e^{P h s} d s=\frac{1}{P h}\left(e^{P h}-1\right), \\
& \bar{H}_{1}=\int_{0}^{1} s e^{P h s} d s=\left(\frac{1}{P h}-\frac{1}{P^{2} h^{2}}\right) e^{P h}+\frac{1}{P^{2} h^{2}}, \\
& \Lambda_{1}=\frac{1}{2} \int_{0}^{1} s^{2} d s=\frac{1}{6}, \\
& a_{1}=1 ; \quad a_{2}=0 ; \quad b_{1}=-1 ; \quad b_{2}=0 ; \\
& \alpha_{1}=1 ; \quad \alpha_{2}=-1 ; \quad \beta_{1}=0 ; \quad \beta_{2}=1 .
\end{aligned}
$$

Similarly, for $n=2$,

$$
\begin{aligned}
& h_{1}(t)=\left\{1+\frac{2}{h}\left(t-t_{1}\right)\right\} \frac{\left(t-t_{2}\right)^{2}}{h^{2}} \\
& h_{2}(t)=\left\{1-\frac{2}{h}\left(t-t_{2}\right)\right\} \frac{\left(t-t_{1}\right)^{2}}{h^{2}} \\
& h_{1}(t)=\frac{1}{h^{2}}\left(t-t_{1}\right)\left(t-t_{2}\right)^{2} \\
& h_{2}(t)=\frac{1}{h^{2}}\left(t-t_{1}\right)^{2}\left(t-t_{2}\right) \\
& \pi(t)=\left(t-t_{1}\right)\left(t-t_{2}\right) .
\end{aligned}
$$

Using $t-t_{2}=h s$, we obtain

$$
\begin{aligned}
& h_{1}(s)=(2 s+3) s^{2}, \\
& h_{2}(s)=(1-2 s)(s+1)^{2}, \\
& h_{1}(s)=(s+1) s^{2}, \\
& h_{2}(s)=(s+1)^{2} s, \\
& \pi(s)=s(s+1) .
\end{aligned}
$$

The simple integration gives the required coefficients. The values of $a_{r}, b_{r}, \alpha_{r}, \beta_{r}$ and $\Lambda_{n}$ are given in Table 1 for $n=O(1) 4$.

5. Numerical Comparison. A simple example

$$
Y^{\prime}(t)=-100 t y^{2}, \quad Y(1)=1 / 51
$$

has been chosen to show the advantages of the present method over the method developed by Norsett [3]. The exact solution of (5.1) is

$$
Y(t)=1 /\left(1+50 t^{2}\right) \text {. }
$$

We have solved (5.1) with the method of Norsett and the present method for different values of $n$ and for different step sizes, on an IBM 360 in single precision. Some of the results are given in Table 2. Our results are closer to the exact solution, even when a smaller number of starting values are used. 
TABLE 1-a

\begin{tabular}{|c|c|c|c|c|c|c|c|c|}
\hline$n \quad i$ & $a_{1}$ & $a_{2}$ & $a_{3}$ & $a_{4}$ & $a_{5}$ & $a_{6}$ & $a_{7}$ & $a_{8}$ \\
\hline 1 & 1 & 0 & & & & & & \\
\hline 1 & 5 & -12 & 18 & -12 & & & & \\
\hline 2 & -4 & 12 & -18 & 12 & & & & \\
\hline 1 & 10 & -33 & 83 & -148.5 & 168 & -90 & & \\
\hline 2 & 9 & -24 & 44 & -48 & 24 & 0 & & \\
\hline 3 & -18 & 57 & -127 & 196.5 & -192 & 90 & & \\
\hline 1 & $\frac{47}{3}$ & $\frac{-550}{9}$ & $\frac{10523}{54}$ & $\frac{-8891}{18}$ & $\frac{8620}{9}$ & $\frac{-12020}{9}$ & $\frac{10740}{9}$ & $\frac{-4620}{9}$ \\
\hline 2 & 64 & -240 & 728 & -1734 & 3120 & -3990 & 3240 & -1260 \\
\hline 3 & -36 & 150 & $\frac{-1009}{2}$ & $\frac{2643}{2}$ & -2580 & 3540 & -3060 & 1260 \\
\hline 4 & $\frac{-128}{3}$ & $\frac{1360}{9}$ & $\frac{-11296}{27}$ & $\frac{8158}{9}$ & $\frac{-13480}{9}$ & $\frac{16070}{9}$ & $\frac{-12360}{9}$ & $\frac{4620}{9}$ \\
\hline
\end{tabular}

TABLE 1-b

$\begin{array}{llllllllll}n & i & b_{1} & b_{2} & b_{3} & b_{4} & b_{5} & b_{6} & b_{7} & b_{8}\end{array}$

$\begin{array}{llll}1 & 1 & -1 & 0\end{array}$

$\begin{array}{llllll}2 & 1 & 0 & 0 & -6 & 12\end{array}$

$\begin{array}{lllll}2 & -1 & 0 & 6 & -12\end{array}$

$\begin{array}{llllllll}3 & 1 & 0 & 0 & -3.5 & 25.5 & -78 & 90\end{array}$

$\begin{array}{lllllll}2 & 0 & 0 & -8 & 24 & -24 & 0\end{array}$

$\begin{array}{lllllll}3 & -1 & 0 & 11.5 & -49.5 & 102 & -90\end{array}$

$\begin{array}{llllllllll}4 & 1 & 0 & 0 & -\frac{8}{3} & \frac{238}{9} & -\frac{400}{3} & \frac{3590}{9} & -\frac{2040}{3} & \frac{1540}{3}\end{array}$

$\begin{array}{lllllllll}2 & 0 & 0 & -13.5 & 121.5 & -540 & 1380 & -1980 & 1260\end{array}$

$\begin{array}{lllllllll}3 & 0 & 0 & 0 & -54 & 360 & -1110 & 1800 & -1260\end{array}$

$4-1 \quad 0 \quad \frac{97}{6} \quad-\frac{1691}{18} \quad \frac{940}{3} \quad-\frac{6020}{9} \quad \frac{2580}{3}-\frac{1540}{3}$ 
TABLE 1-C

\begin{tabular}{rrrrrrrrrrr}
$n$ & $i$ & $\alpha_{1}$ & \multicolumn{1}{c}{$\alpha_{2}$} & \multicolumn{1}{c}{$\alpha_{3}$} & \multicolumn{1}{c}{$\alpha_{4}$} & $\alpha_{5}$ & $\alpha_{6}$ & $\alpha_{7}$ & $\alpha_{8}$ & $\Lambda_{n}$ \\
1 & 1 & 1 & -1 & & & & & & & $1 / 3$ \\
2 & 1 & 2 & -5 & 8 & -6 & & & & & $31 / 30$ \\
& 2 & 4 & -8 & 10 & -6 & & & & & \\
3 & 1 & 3 & -10 & 25.5 & -46.5 & 54 & -30 & & & $\frac{869}{105}$ \\
& 2 & 18 & -57 & 136 & -228 & 240 & -120 & & & \\
& 3 & 9 & -24 & 48.5 & -70.5 & 66 & -30 & & & \\
4 & 1 & 4 & $-\frac{47}{3}$ & $\frac{452}{9}$ & $-\frac{769}{6}$ & $\frac{752}{3}$ & $-\frac{1060}{3}$ & 320 & -140 & $\frac{74113}{630}$ \\
& 2 & 48 & -184 & 574 & -1416 & 2658 & -3570 & 3060 & -1260 & \\
& 3 & 72 & -264 & 781 & -1813.5 & 3192 & -4020 & 3240 & -1260 & \\
& 4 & 16 & $-\frac{152}{3}$ & $\frac{1178}{9}$ & $-\frac{1624}{6}$ & $\frac{1298}{3}$ & $-\frac{1510}{3}$ & 380 & -140 &
\end{tabular}

TABLe 1-d

$$
\begin{array}{rrrrrrrrrr}
n & i & \beta_{1} & \beta_{2} & \beta_{3} & \beta_{4} & \beta_{5} & \beta_{8} & \beta_{7} & \beta_{8} \\
1 & 1 & 0 & 1 & & & & & & \\
2 & 1 & 0 & 0 & -2 & 6 & & & & \\
& 2 & 0 & 1 & -4 & 6 & & & & \\
3 & 1 & 0 & 0 & -1 & \frac{15}{2} & -24 & 30 & & \\
& 2 & 0 & 0 & -8 & 48 & -120 & 120 & & \\
& 3 & 0 & 1 & -6 & \frac{39}{2} & -36 & 30 & & \\
4 & 1 & 0 & 0 & \frac{-2}{3} & \frac{20}{3} & -34 & \frac{310}{3} & -180 & 140 \\
& 2 & 0 & 0 & -9 & \frac{171}{2} & -408 & 1140 & -1800 & 1260 \\
& 3 & 0 & 0 & -18 & 144 & -582 & 1410 & -1980 & 1260 \\
4 & 0 & 1 & \frac{-22}{3} & \frac{193}{6} & -96 & \frac{580}{3} & -240 & 140
\end{array}
$$


TABLE 2

$\begin{array}{ccccc}h & Y(t) & \begin{array}{c}\text { Norsett's Method } \\ n=3\end{array} & \begin{array}{c}\text { Present Method } \\ n=2\end{array} & \text { Exact } \\ & & 1 & n=2 & \\ \frac{1}{16} & Y(10) & .19995554(-3) & .19996018(-3) & .19996001(-3) \\ & Y(10) & .19991486(-3) & .19996310(-3) & .19996001(-3) \\ \frac{1}{8} & Y(20) & .49994562(-4) & .49997695(-4) & .49997500(-4) \\ & Y(10) & .19906134(-3) & .20000938(-3) & .19996001(-3) \\ \frac{1}{4} & Y(20) & .49940176(-4) & .50000607(-4) & .49997500(-4)\end{array}$

6. Remarks. In order to apply (2.3), we need to know the first derivative of $f(t, y)$. If the exact derivative is not available, the numerical differentiation may be used. But in such a case, we are not sure whether the order of this method is preserved or not. It is also hoped that some generating functions and recurrence relations may be derived to obtain $H_{i}$, and $\bar{H}_{i}$ directly.

7. Acknowledgments. The author wishes to thank the referee for his suggested improvements of this paper.

Department of Mathematics

University of Saskatchewan

Saskatchewan, Saskatoon, Canada

1. G. G. Dahlquist, "A special stability problem for linear multistep methods," Nordisk Tidskr. Informations-Behandling, v. 3, 1963, pp. 27-43. MR 30 \#15.

2. C. E. TREANOR, "A method for the numerical integration of coupled first-order differential equations with greatly different time constants," Math. Comp., v. 20, 1966, pp. 39-45. MR 33 \#889.

3. S. P. Norsetr, "An $A$-stable modification of the Adams-Bashforth methods," Conference on the Numerical Solution of Differential Equations (Dundee, Scotland, June 1969).

4. A. Ralston, A First Course In Numerical Analysis, McGraw-Hill, New York, 1965. MR 32 \#8479.

5. C. W. Gear, Numerical Integration of Stiff Ordinary Differential Equations, Report \#221, University of Illinois, Department of Computer Science, January 1967.

6. P. HENRICI, Discrete Variable Methods In Ordinary Differential Equations, Wiley, New York, 1962. MR 24 \#B1772. 